\title{
The Chinese medicine formula HB01 reduces choroidal neovascularization by regulating the expression of vascular endothelial growth factor
}

\author{
Ming Jin ${ }^{1 *}$, Youhua Zhang ${ }^{1}$, Lin Pan ${ }^{1}$, Renhui Dou' ${ }^{1}$, Robert B Nussenblatt ${ }^{2,3}$ and Lai Wei ${ }^{2,3^{*}}$
}

\begin{abstract}
Background: Choroidal neovascularization (CNV) remains the leading cause of newly acquired blindness in the developed world. Currently anti-vascular endothelial growth factor (VEGF) therapies are broadly used to treat neovascular ocular disorders. Here we demonstrate the effect of a traditional Chinese medicine formula, HB01, on CNV.
\end{abstract}

Methods: A rat model of laser-induced CNV was used to investigate the effect of HB01 in vivo. The CNV lesions in the eye were evaluated using fundus fluorescein angiography and visualized/quantified using confocal microscopy. Expression of VEGF in the choroidal and retinal tissues was measured using quantitative real-time PCR and immunohistochemistry.

Results: We demonstrated that a traditional Chinese Medicine formula, named HB01, significantly reduced neovascularization in a rat CNV model. The effect of HBO1 on CNV was comparable to the intravitreal injection of bevacizumab (Avastin). Our results also suggested that HBO1 may reduce CNV partially through inhibiting the expression of VEGF.

Conclusions: These data support HB01 as an alternative therapy for ocular neovascular disorders.

Keyword: Choroidal neovascularization, Traditional Chinese medicine, VEGF

\section{Background}

Many ocular diseases, such as the wet or exudative/neovascular age-related macular degeneration (AMD), myopia and diabetic retinopathy, involve in choroidal neovascularization $(\mathrm{CNV})$, which remains the leading cause of newly acquired blindness in the developed world [1]. In neovascular AMD, blood vessels compromise Bruch's membrane from the choroid and grow into the space beneath the retinal pigment epithelium (RPE), or within the subretinal space between RPE and photoreceptor. The loss of visual acuity is caused by serum or blood leaked from the proliferating blood vessels underneath and within the retina. A subretinal fibrovascular scar eventually develops if the CNV is left untreated, leading to a permanent loss of vision [2]. On the other hand, in proliferative diabetic retinopathy, neovascularization

\footnotetext{
* Correspondence: jinming321@yahoo.com.cn; lai.wei@nih.gov

'Department of Ophthalmology, China-Japan Friendship Hospital, Beijing University of Chinese Medicine, Beijing, China

Full list of author information is available at the end of the article
}

originates in the retinal vessels and appears on the surface of the retina. These leaky blood vessels can lead to hemorrhages and fluid in the retinal tissue and vitreous, which compromises vision [3].

Several treatments for CNV are currently available, with variable efficacy on disease progress, including local corticosteroids, submacular surgical removal of CNV, laser photocoagulation, and photodynamic therapies [4,5]. More recently, the development of drugs directly suppressing the growth and development of blood vessels provides new outlooks for effectively controlling CNV. A number of large randomized clinical trials suggest that vascular endothelial growth factor (VEGF) is a central player in pathogenesis of $\mathrm{CNV}$ and inhibiting its function locally in the eye is sufficient to cause a decrease in angiogenesis that leads to a relief of symptoms in wet AMD and other neovascular ocular diseases. Among all anti-VEGF therapeutic agents, the anti-VEGF antibody drugs ranibizumab (Lucentis, Genentech) and bevacizumab (Avastin, Genentech) are most broadly 
used to treat AMD with similar efficacy and side effects $[6,7]$.

Although current therapeutic options to treat $\mathrm{CNV}$ are effective for the majority of patients, we have been looking for more successful and definitive alternatives with improved efficacy, reduced cost, and less complications. A rich literature including ancient Chinese medical records and modern Chinese studies in the past decades demonstrates some success of traditional Chinese medicine treating neovascular ocular diseases such as wet AMD [8-10]. We have previously shown that a traditional Chinese medicine formula, HB01, can reduce $\mathrm{CNV}$ and hemorrhage in the eyes as well as improve the visual acuity in patients with neovascular AMD, pathological myopia, or central exudative chorioretinitis [11]. HB01 is the water extract of six herbs including Astragalus membranaceus, Angelica sinensis, Panax Notoginseng, Pollen Typhae, Bulbus Fritillariae Thunbergii, Citrus reticulata, in the ratio of 1:1:1:1:1:1 (9 g each). The major functional component of HB01, Astragalus membranaceus, has been used for thousands of years in Asia to prevent cold and respiratory infections. It has both anti-bacterial and anti-inflammatory effects [12]. While Angelica sinensis, Panax Notoginseng, and Pollen Typhae can stop bleeding [13-15], Citrus reticulata has been used to relieve congestion in the lung [16]. Therefore, HB01 has both anti-inflammatory and anti-hemorrhage effects. Our current study is to investigate whether HB01 can limit the formation of $\mathrm{CNV}$ and the molecular mechanism underlying its effect in a laser-induced CNV rat model.

\section{Methods}

HB01

HB01 is comprised of six herbs, Astragalus membranaceus, Angelica sinensis, Panax Notoginseng, Pollen Typhae, Bulbus Fritillariae Thunbergii, Citrus reticulate, in the ratio of 1:1:1:1:1:1 (9 g each), respectively. The water extract (total of $500 \mathrm{ml}$ per day) of mixture of all herbs was prepared and packed by the department of Pharmaceutical Sciences, China-Japan Friendship Hospital, Beijing University of Chinese Medicine (Beijing, China) using standardized procedure.

\section{Laser-induced CNV in rats}

Eight week old male Brown Norway rats weighing between 200 and $250 \mathrm{~g}$ were purchased from Vital River Inc. (Beijing, China) and maintained in accordance with the Institutional Animal Care and Use Committee procedures and guidelines, as well as ARVO guidelines on the use of Animals in Ophthalmic and Vision Research. The CNV was induced as previously described $[17,18]$. Briefly, rats were anesthetized by intraperitoneal injection of ketamine $(70 \mathrm{mg} / \mathrm{kg})$ and xylazine $(7 \mathrm{mg} / \mathrm{kg})$. The left pupil was dilated with 1 drop of $1 \%$
Tropicamide (Beijing Double-Crane Pharmaceutical Business Co., Beijing, China). Then the left eye received 10-12 laser burns between retinal vessels around the optic nerve head ( $360 \mathrm{~mW}, 50 \mathrm{~ms}, 0.05 \mathrm{~mm}$ spot size) using an ARAMIS II Argo Laser (Quantel Medical, France). The rupture of Bruch's membrane was confirmed in all laser lesions. Animals were divided into 4 groups and treated as following: 1) Control group did not received laser or drug treatment, and was given $2 \mathrm{ml} / 0.2 \mathrm{~kg}$ PBS once daily by oral gavage starting on Day 0 for 4 weeks; 2) $C N V$ group received laser on Day 7 but not drug treatment, and was given $2 \mathrm{ml} / 0.2 \mathrm{~kg}$ PBS once daily by oral gavage starting on Day 0 for 4 weeks; 3) CNV with bevacizumab group received laser on Day 7 and one intravitreal injection (25 ug in 1 ul solution, on Day 14) of bevacizumab (Roche, USA); 4) $C N V$ with $H B 01$ group received laser on Day 7 and HB01 (once daily by oral gavage, $2 \mathrm{ml} / 0.2 \mathrm{~kg}$, starting on Day 0 for 4 weeks). The dose of HB01 used in rats was calculated according to the one used in our previous study in AMD patients $(500 \mathrm{ml} / 50 \mathrm{~kg} /$ day $)$ [11].

\section{Fundus fluorescein angiography}

CNV lesions were evaluated by fundus fluorescein angiography as previously described [18]. On Day 7, 14, 21, and 28 after laser photocoagulation, the CNV lesions were visualized using a digital fundus camera (Topcon, Japan). The mean area of CNV was calculated using Image-Pro Plus 6.0 (Kodak, USA) by two independent ophthalmologists blinded to the experimental design.

\section{Visualizing and quantifying CNV}

$\mathrm{CNV}$ was visualized and quantified as previously described [18]. Briefly, rats were anesthetized, killed with $100 \% \mathrm{CO} 2$, and perfused through the left ventricle injection of $1 \mathrm{ml}$ PBS, followed by perfusion of lactated Ringer's solution (Abbott Laboratories, USA) containing 10\% gelatin with $5 \mathrm{mg} / \mathrm{ml}$ FITC-Dextran (Sigma, USA). The eyes were removed and fixed in 10\% formalin for 1 hour. The sclera-choroid-RPE complex was flat-mounted after removing the cornea, lens and the neurosensory retina. Flat mounts were visualized using a confocal microscope (Zeiss LSM510). Images of the neovascular lesions were captured using a digital camera (OlympusBX-51) and analyzed using Image-Pro Plus 6.0 (Kodak, USA). A laser spot with green vessels were scored $\mathrm{CNV}$ positive, while a laser spot lacking green vessels were scored CNV-negative.

\section{Histopathology and Immunohistochemistry}

On Day 28, the eyes were enucleated and fixed in $4 \%$ paraformaldehyde overnight. The posterior eye cup was embedded in paraffin and serial sections of $4 \mu \mathrm{m}$ thick were cut and used for either H\&E staining or immunostaining. To measure the thickness of $\mathrm{CNV}$ lesions, the sections 

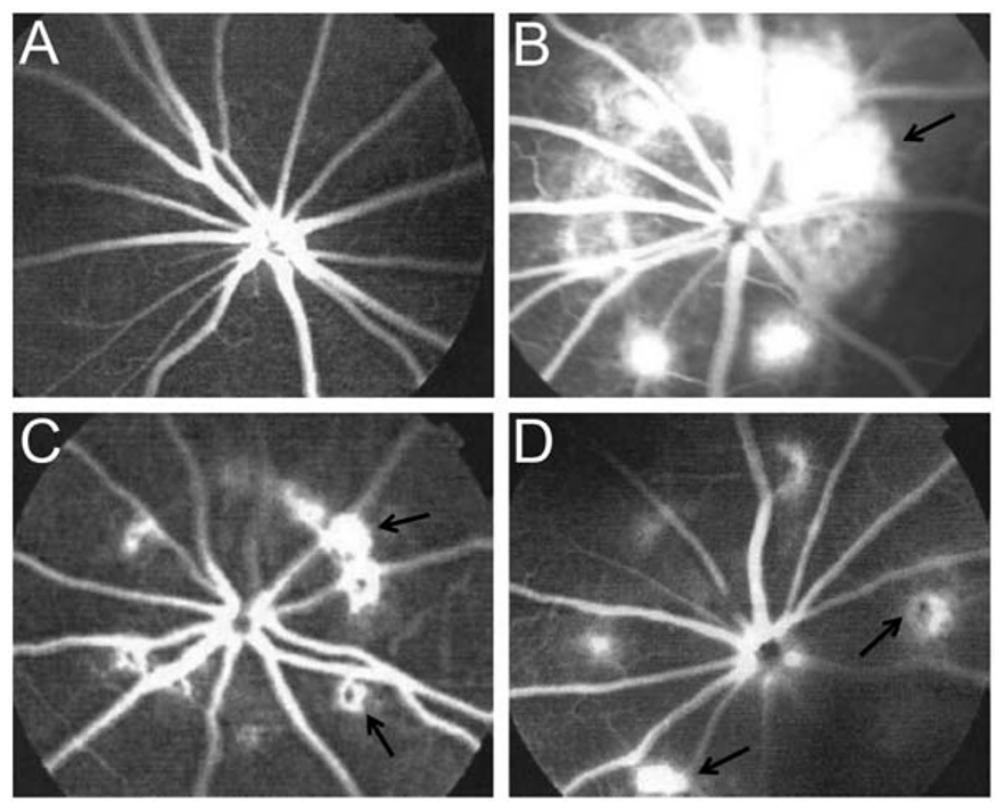

Figure 1 Fundus fluorescein angiography. (A) Fundus fluorescein angiography of a representative rat in the Control group that did not received laser or drug treatment. (B) Fundus fluorescein angiography of a representative rat in the CNV group that received laser on Day 7 but not drug treatment. (C) Fundus fluorescein angiography of a representative rat in the CNV with bevacizumab group that received laser on Day 7 and one intravitreal injection of bevacizumab on Day 14. (D) Fundus fluorescein angiography of a representative rat in the CNV with HB01 group received laser on Day 7 and HB01 treatment for four weeks. Arrows marked lesions.

with the largest areas of the CNV lesions were selected. The maximum thickness of the selected CNV lesion centers were recorded by a masked pathologist. The rabbit polyclonal anti-VEGF antibody and rabbit anti-human IgG (Wuhan Boster Bio-Engineering LLC, China) were used in immunohistochemistry analysis.

\section{RNA isolation and quantitative real-time PCR}

The retinal and choroidal tissue was dissected from the rat eyes on Day 28 and RNA was extracted using TRIzol reagent (Invitrogen, USA). The reverse transcription was performed using PrimeScript RT reagent Kit (TaKaRa Bio Inc, Japan) according to the protocol manufacture provided. Real-time PCR was performed with SYBR Green PCR Master Mix on the ABI PRISM 7500 Sequence Detection System (Applied Biosystems, USA). The relative expression of Vegfa was normalized to Actb. The following primer sets were used:

Actb-F: CCTGTATGCCTCTGGTCGTA, Actb-R: TGAGCTATGAAGGCGACGTTACCA; Vegfa-F: CTCACCAAAGCCAGCACATAGGAGAG, Vegfa-R: TCTGCGGATCTTGGAC-AAACAAATGC.

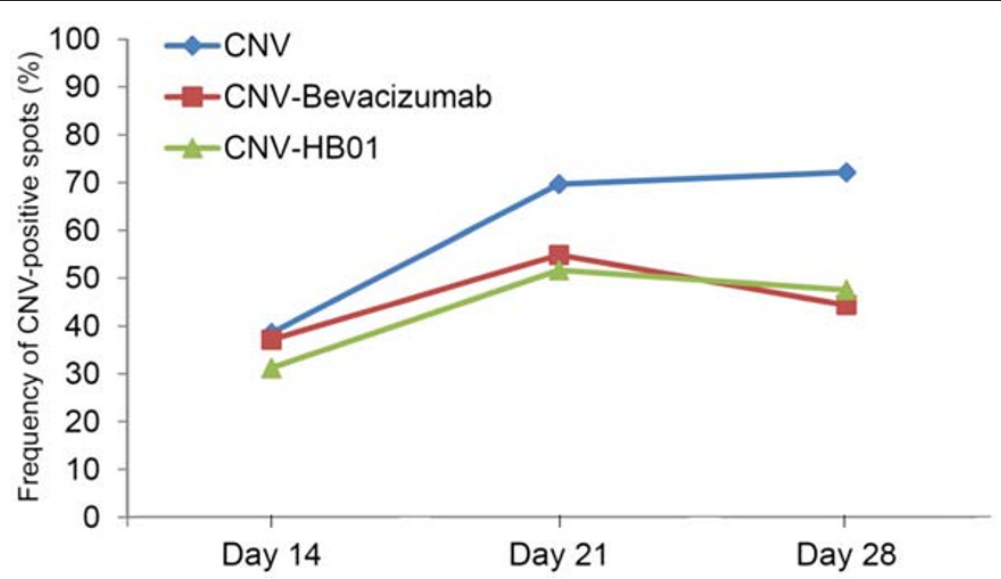

Figure 2 The frequency of CNV-positive spots. The CNV lesions were evaluated on Day 14, Day 21, and Day 28. The percentage of CNV positive spots was calculated as the ratio of CNV positive spots (in all eyes within a group of rats) and all CNV lesions. 


\section{Statistical analysis}

All data were analyzed using GraphPad Prism 5.0 (GraphPad Software, USA). Statistical significance was determined by Mann-Whitney test or Chi-square test as indicated in the figure legend. A P value less than 0.05 is considered statistically significant.

\section{Results}

Prevention of CNV formation by $\mathrm{HB} 01$

To determine the effect of HB01 on laser induced CNV, we first carried out fundus fluorescein angiography. Rats were divided into four groups (12 rats in each group) as indicated in the Methods Section. The CNV was
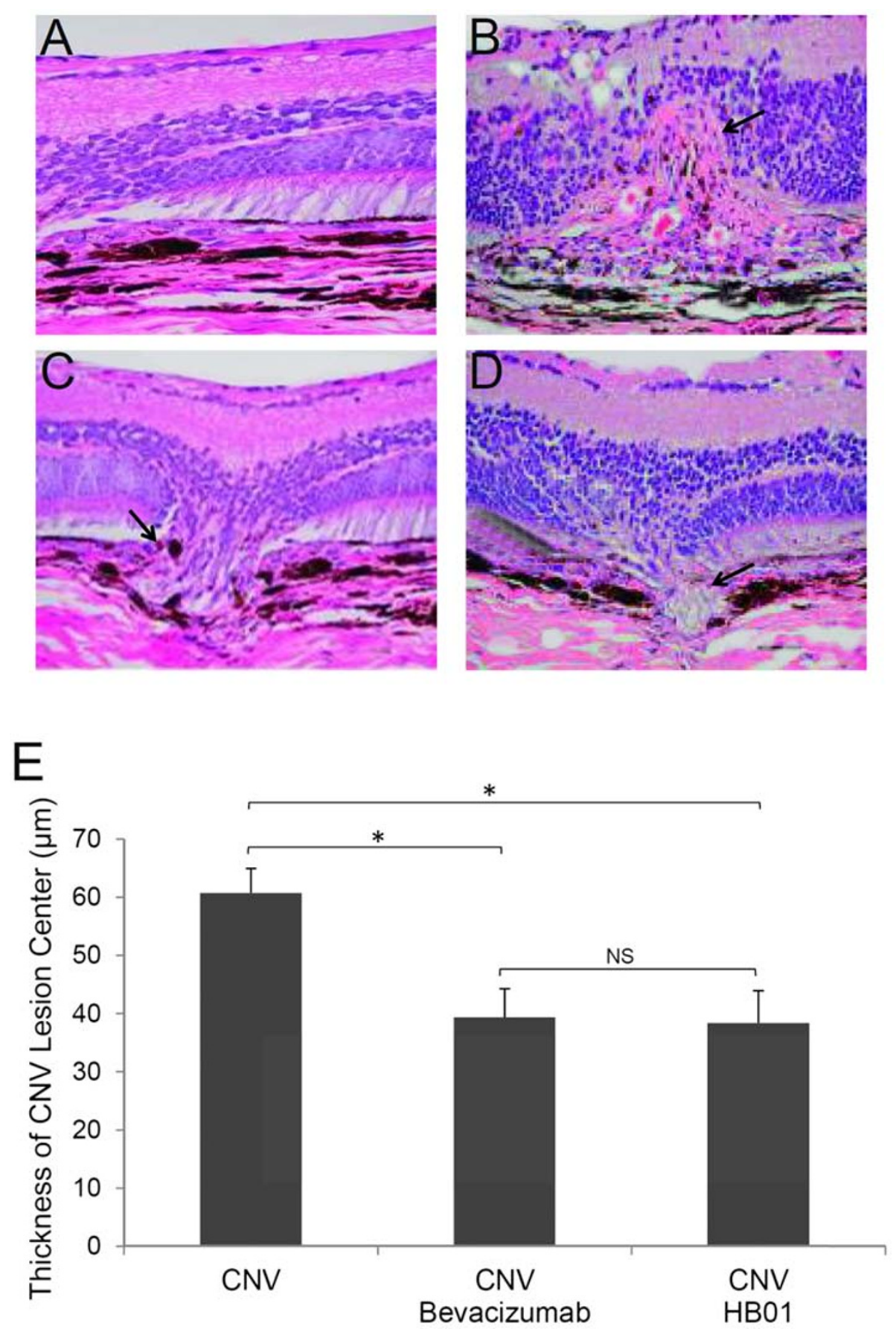

Figure 3 Histologic analysis of CNV lesions in rat eyes. Paraffin-embedded cross sections through CNV lesions were examined using Hematoxylin/eosin (H\&E) staining for eyes from the Control (A), CNV (B), CNV with bevacizumab (C), and CNV with HB01 (D) groups on Day 28. The thickness of the retinal lesion centers was summarized in (E). ${ }^{*} \mathrm{P}<0.01$, Mann Whitney test; NS, not significant. Arrows marked CNV lesions. 

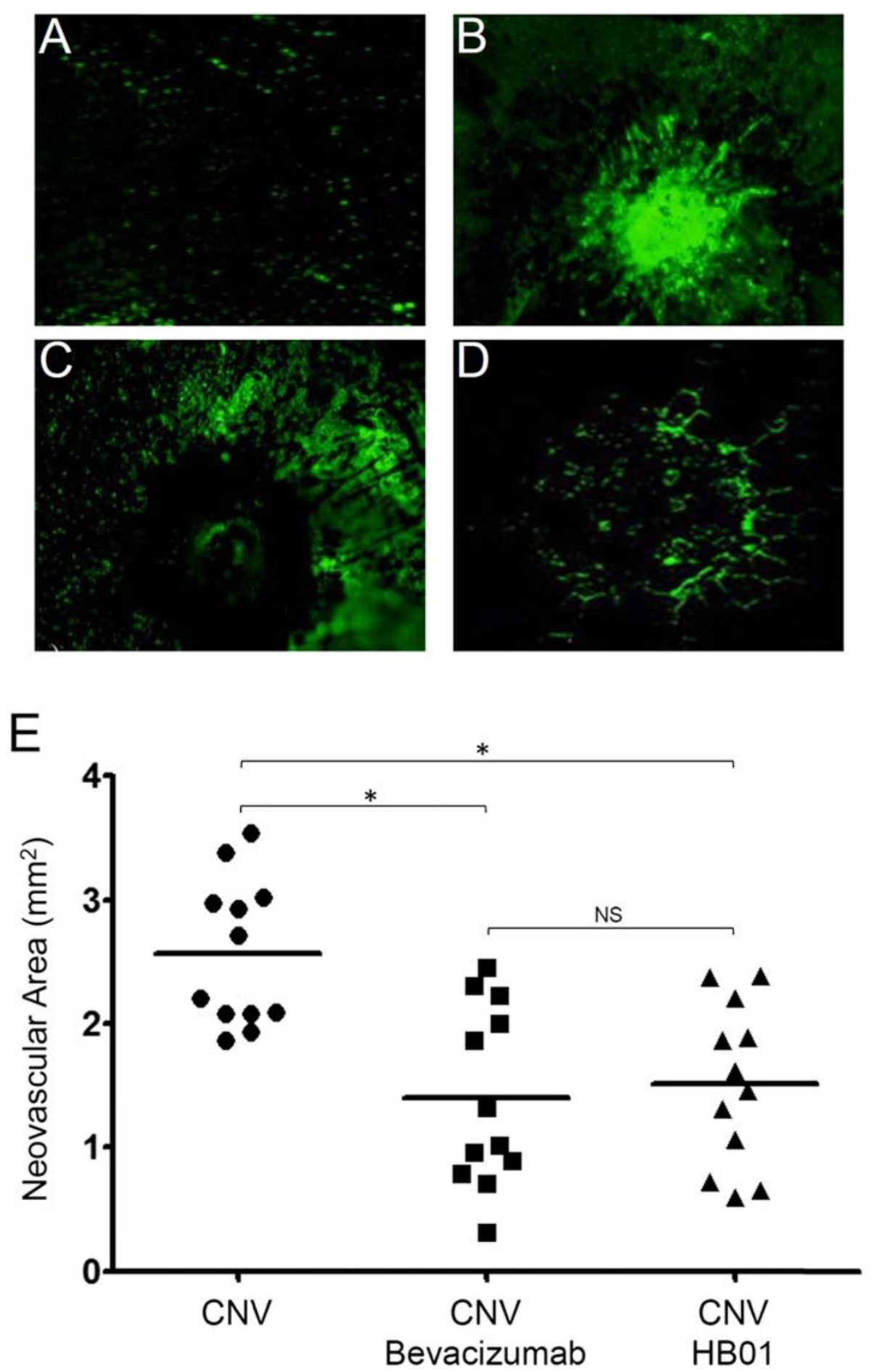

Figure 4 Confocal microscopy of CNV lesions. The fluorescent images of CNV in RPE-choroid-sclera flat mounts from the Control (A), CNV (B), CNV with bevacizumab (C), and CNV with HB01 (D) groups were shown. The vasculature was labeled with FITC-dextran via cardiac perfusion on Day 28. The total sizes of neovascular areas for eyes from CNV, CNV with bevacizumab, and CNV with HB01 were summarized in (E). *P $<0.01$, Mann Whitney test; NS, not significant. 
evaluated on Day 14, 21, and 28 after laser induction of CNV spots on Day 7. As shown in Figure 1, robust choroidal neovascular lesions formed in lasered, PBS treated rats (Figure $1 \mathrm{~B}$ ) on Day 28, while rats treated with both bevacizumab (Figure 1C) and HB01 (Figure 1D) appeared to have fewer and smaller lesions. To quantify the incidence of CNV formation, we calculated the percentage of CNV-positive spots among all lesion spots. On Day 14, the numbers of CNV-positive spots for all three groups receiving laser were not different (31-38\%) (Figure 2). However, interestingly, both bevacizumab and HB01 significantly reduced the numbers of CNVpositive spots in the rat eyes on Day 21 and 28 (Figure 2). No difference was found in regression of $\mathrm{CNV}$ between bevacizumab and HB01 groups. In addition, we performed histologic analysis of paraffin-embedded cross section of the rat retinal tissues. As shown in Figure 3, laser induced damage of retinal and choroidal structure and broad neovascularization in untreated rats (Figure 3B), as compared to the normal eyes (Figure 3A). Both bevacizumab (Figure 3C) and HB01 (Figure 3D) reduced neovascularization and partially restored the normal retinal and choroidal structure. In addition, both treatments significantly reduced the thickness of retinal lesions caused by CNV (Figure 3E). These results indicate that the Chinese medicine formula HB01 may induce regression of laser-induced $\mathrm{CNV}$, similar to the effect of intravitreal injection of bevacizumab.

To further determine whether HB01 can decrease neovascularization, we measured the size of $\mathrm{CNV}$ in the images of FITC-dextran labeled RPE-choroid-sclera flat mounts. On Day 28, an area of hyperfluorescence with a diameter approximately equal to that of the laser spot was found only in the eyes received laser but no drug treatment (Figure 4B), while both bevacizumab (Figure 4C and 4E) and HB01 (Figure 4D and significantly reduced the size of laser4E) significantly reduced the size of laser-induced CNV formation. Taken together, our results suggest a significant effect of the Chinese medicine formula $\mathrm{HB} 01$ on reduction of $\mathrm{CNV}$ in vivo.

\section{Expression of VEGF regulated by $\mathrm{HB} 01$}

To investigate the potential molecular mechanism underlying the effect of HB01 on CNV formation, we performed quantitative Real-time PCR and immunohistochemistry analysis to evaluate the expression of VEGF, the potent angiogenic factor previously shown to be responsible for promoting CNV. Consistent with previous studies [19], intravitreal injection of bevacizumab significantly reduced expression of Vegfa mRNA (Figure 5) and its protein (Figure $6 \mathrm{C}$ and $6 \mathrm{E}$ ) in lasered eyes, as compared to the one received no drug treatment (Figure 5 and Figure $6 \mathrm{~B}$ and $6 \mathrm{E}$ ). Intriguingly, oral administration of $\mathrm{HBO} 1$ also resulted in a significantly

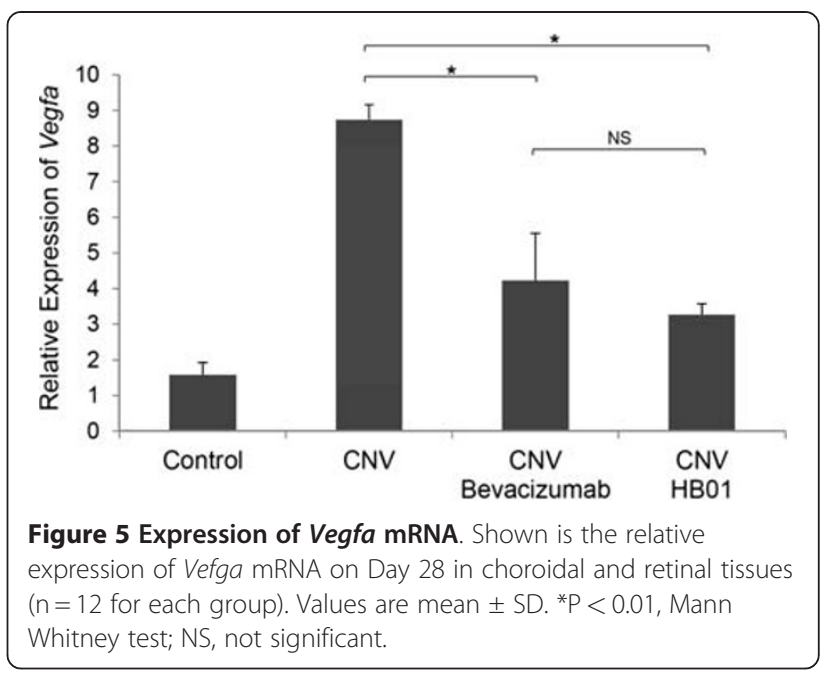

reduction of Vegfa mRNA (Figure 5) and its protein (Figure 6D and 6E) in lasered eyes, as compared to the one received no drug treatment (Figure 5 and Figure $6 \mathrm{~B}$ and $6 \mathrm{E})$. These results suggest that HB01 may function to reduce CNV by controlling the expression of VEGF.

\section{Discussion}

Laser induced CNV model has been established in both non-human primates and rodents [20-22]. It shows remarkable similarity to the pathology found in many neovascular ocular disorders such as wet AMD. It has been extensively used to evaluate the efficacy of anti-CNV therapies [1]. In our study, we demonstrated that a traditional Chinese Medicine formula, named HB01, significantly reduced neovascularization in a rat $\mathrm{CNV}$ model. The effect of HB01 on CNV was comparable to the intravitreal injection of bevacizumab (Avastin). Our results also suggested that $\mathrm{HB} 01$ may reduce CNV partially through inhibiting the expression of VEGF. To our knowledge, this is the first attempt to understand whether and how traditional Chinese medicine can reduce $\mathrm{CNV}$ in a rodent model.

Current therapeutic strategies for $\mathrm{CNV}$ often involve in invasive approaches such as intravitreal injection or surgery. However, none of these treatments fully restore the lost vision in most patients and they can result in complications leading to further vision loss $[23,24]$. The oral administration of traditional Chinese herbal medicine could provide an alternative but effective approach for CNV therapy. Often time, thousands of years of clinical experiments have optimized formulas in Chinese medicine. Therefore, it will be reasonable to expect that most of the classical formulas work to some extent as indicated in the ancient literature. The formula HB01 exemplifies the expectation and shows its effectiveness in reducing $\mathrm{CNV}$ in a small randomized clinical trial [11]. However, our current and further studies aim to 

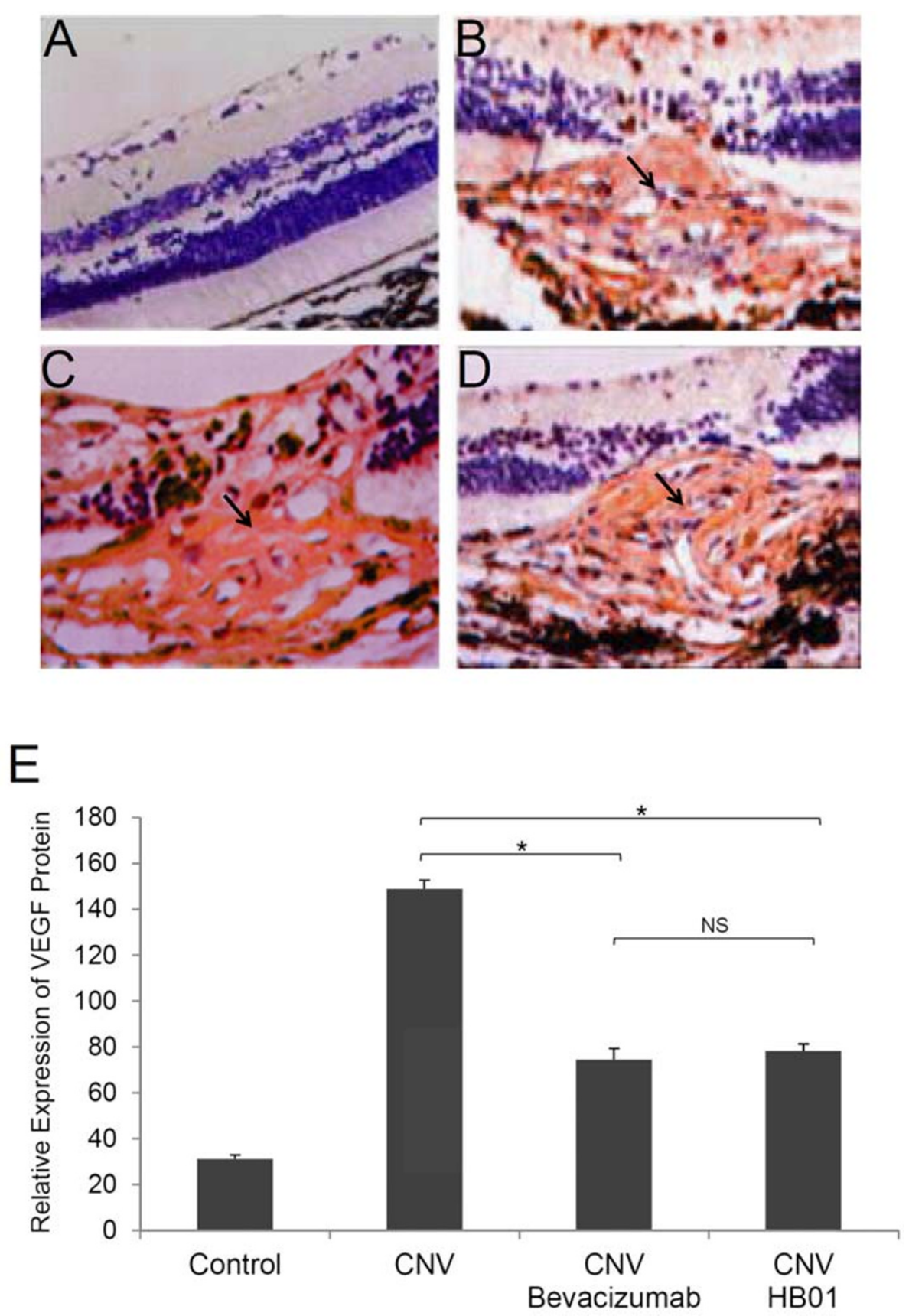

Figure 6 Expression of VEGF protein. Shown is the immunostaining of VEGF in the CNV lesions on Day 28 in eyes from the Control (A), CNV (B), CNV with bevacizumab (C), and CNV with HB01 (D) groups. The relative expression of VEGF protein was summarized in (E). ${ }^{*} \mathrm{P}<0.01$, Mann Whitney test; NS, not significant. Arrows marked CNV lesions.

elucidate the molecular mechanism by which HB01 reduces $\mathrm{CNV}$. In addition, a therapy combining both HB01 and anti-VEGF treatment will be especially interesting, which could improve the efficacy and safety as well as limit the complications associate with the therapy.

Currently, there is a great debate about use of Chinese herbal medicine as a sole treatment for diseases, considering its potential placebo-only effect [25]. Moreover, producing an herbal formula with constant active ingredients remains a great challenge [26]. In addition, the laser-induced rat CNV model involves a wound healing process that is potentially different from the pathogenesis of wet AMD. It is necessary to investigate the effect of HB01 on other AMD animal models. Moreover, 
further study is warranted to characterize chemical composition of HB01 and its drug targets in vitro and in vivo.

\section{Conclusions}

Our results demonstrate that $\mathrm{HB} 01$ reduced $\mathrm{CNV}$ in a rat model of laser-induced $\mathrm{CNV}$, probably by inhibiting the expression of VEGF. This data support HB01 as an alternative therapy for ocular neovascular disorders.

\section{Competing interests}

MJ has a patent (CN102058845A) on the HB01 used in this study.

\section{Authors' contributions}

$M J$ and $Y Z$ designed the study. YZ, LP, and RD carried out experiments. MJ and LW performed statistical analysis. MJ, RBN, and LW participated in the design of study, interpretation of results, and drafted the manuscript. All authors read and approved the final manuscript.

\section{Acknowledgements}

This work was supported by a research grant from the National Natural Science Foundation of China awarded to Dr. Jin (30973775) and by the intramural research program of National Eye Institute and National Center for Complementary and Alternative Medicine, NIH, USA. We thank Dr. C. Chan for critical reading of the manuscript.

\section{Author details}

'Department of Ophthalmology, China-Japan Friendship Hospital, Beijing University of Chinese Medicine, Beijing, China. 'Laboratory of Immunology, National Eye Institute, National Institutes of Health, Bethesda, MD, USA. ${ }^{3}$ National Center for Complementary and Alternative Medicine, National Institutes of Health, Bethesda, MD, USA.

Received: 17 December 2011 Accepted: 26 April 2012

Published: 7 June 2012

\section{References}

1. Andreoli CM, Miller JW: Anti-vascular endothelial growth factor therapy for ocular neovascular disease. Curr Opin Ophthalmol 2007, 18(6):502-508

2. Folk JC, Stone EM: Ranibizumab therapy for neovascular age-related macular degeneration. N Engl J Med 2010, 363(17):1648-1655.

3. Caldwell RB, et al: Vascular endothelial growth factor and diabetic retinopathy: pathophysiological mechanisms and treatment perspectives. Diabetes Metab Res Rev 2003, 19(6):442-455.

4. Caputo $M$, et al: Perspectives of choroidal neovascularization therapy. Curr Drug Targets 2011, 12(2):234-242.

5. Berger AS, et al: Submacular surgery for subfoveal choroidal neovascular membranes in patients with presumed ocular histoplasmosis. Arch Ophthalmol 1997, 115(8):991-996.

6. Martin DF, et al: Ranibizumab and bevacizumab for neovascular agerelated macular degeneration. N Engl J Med 2011, 364(20):1897-1908.

7. van der Reis Ml, et al: A systematic review of the adverse events of intravitreal anti-vascular endothelial growth factor injections. Retina 2011 31(8):1449-1469.

8. Liang XC, et al: Therapeutic efficacy of Stephania tetrandra S. Moore for treatment of neovascularization of retinal capillary (retinopathy) in diabetes-in vitro study. Phytomedicine 2002, 9(5):377-384.

9. Wang $X$, Hu S: A review of research actuality on age-related macular degeneration. Yan Ke Xue Bao 2001, 17(4):245-251.

10. Ming Jin, Zhang YouHua, Haidan Liu: The effect of traditional chinese medicine hexuemingmu on macular hemorrhage. China Journal of Chinese Ophthalmology 2010, 20(2):540-543.

11. Ming Jin, Zhang YouHua, Yanan Qiu: Clinical study on choroidal neovascularization treated with traditional chinese medicine Huangbanbianxing Formula (HB01). Chin J Integr Med 2009, 29(6):540-543.

12. University of Maryland Medical Center:, [http://www.umm.edu/altmed/ articles/astragalus-000223.htm].

13. Ng TB: Pharmacological activity of sanchi ginseng (Panax notoginseng). J Pharm Pharmacol 2006, 58(8):1007-1019.
14. Yi L, et al: The analysis of Radix Angelicae Sinensis (Danggui). J Chromatogr A 2009, 1216(11):1991-2001.

15. Ohkura N, et al: Experimental study on the hemostatc activity of Pollen Typhae: a traditional folk medicine used by external and oral application. Blood Coagul Fibrinolysis 2011, 22(8):631-636.

16. Ho SC, Lin CC: Investigation of heat treating conditions for enhancing the anti-inflammatory activity of citrus fruit (Citrus reticulata) peels. J Agric Food Chem 2008, 56(17):7976-7982.

17. Edelman JL, Castro MR: Quantitative image analysis of laser-induced choroidal neovascularization in rat. Exp Eye Res 2000, 71(5):523-533.

18. Kinose F, et al: Inhibition of retinal and choroidal neovascularization by a novel KDR kinase inhibitor. Mol Vis 2005, 11:366-373.

19. Kim IT, et al: Anti-angiogenic Effect of KR-31831 on Corneal and Choroidal Neovascularization in Rat Models. Invest Ophthalmol Vis Sci 2012, [Epub ahead of print].

20. Ryan SJ: The development of an experimental model of subretinal neovascularization in disciform macular degeneration. Trans Am Ophthalmol Soc 1979, 77:707-745.

21. Dobi ET, Puliafito CA, Destro M: A new model of experimental choroidal neovascularization in the rat. Arch Ophthalmol 1989, 107(2):264-269.

22. Tobe T, et al: Targeted disruption of the FGF2 gene does not prevent choroidal neovascularization in a murine model. Am J Pathol 1998, 153 (5):1641-1646.

23. Jager RD, Mieler WF, Miller JW: Age-related macular degeneration. N Engl J Med 2008, 358(24):2606-2617.

24. Lima LH, et al: Evaluation of safety for bilateral same-day intravitreal injections of antivascular endothelial growth factor therapy. Retina 2009, 29(9):1213-1217.

25. Staud R: Effectiveness of CAM therapy: understanding the evidence. Rheum Dis Clin North Am 2011, 37(1):9-17.

26. Wang $X$, Chan AW: Challenges and patenting strategies for Chinese herbal medicine. Chin Med 2010, 5:24.

doi:10.1186/1479-5876-10-118

Cite this article as: Jin et al:: The Chinese medicine formula HBO1 reduces choroidal neovascularization by regulating the expression of vascular endothelial growth factor. Journal of Translational Medicine 2012 10:118.

\section{Submit your next manuscript to BioMed Central and take full advantage of:}

- Convenient online submission

- Thorough peer review

- No space constraints or color figure charges

- Immediate publication on acceptance

- Inclusion in PubMed, CAS, Scopus and Google Scholar

- Research which is freely available for redistribution 